УДК 621.391:621.396.96

\title{
ОБНАРУЖЕНИЕ-ИЗМЕРЕНИЕ КОГЕРЕНТНО-ИМПУЛЬСНЫХ СИГНАЛОВ
}

\author{
ПОПОВ Д. И.
}

\author{
Рязанский государственный радиотехнический университет, \\ Россия, Рязань, 390005, ул. Гагарина, д. 59/1
}

\begin{abstract}
Аннотация. Синтезированы алгоритмы совместного обнаружения-измерения когерентно-импульсных сигналов с линейной перестройкой параметров, позволяющие однозначно измерять радиальную скорость цели в заданном диапазоне при сохранении однозначного измерения дальности. Предложена структурная схема обнаружителя-измерителя. Моделированием на ЭВМ проведен сравнительный анализ синтезированных и известных алгоритмов обнаружения-измерения
\end{abstract}

Ключевые слова: алгоритм обнаружения-измерения; метод максимального правдоподобия; моделирование алгоритмов обнаружения-измерения; отношение правдоподобия; синтез алгоритмов обнаружения-измерения; точность измерения; характеристики обнаружения

\section{ВВЕДЕНИЕ}

При измерении координат движущихся целей в импульсных радиолокационных системах (РЛС) возникает известная проблема совместного однозначного измерения дальности и радиальной скорости цели [1]. Предел однозначного измерения обеих координат определяется величиной периода повторения $T$ зондирующих импульсов. При проектировании РЛС первоначально предпочтение отдается одной из координат. Для однозначного измерения радиальной скорости цели с высокими разрешающей способностью и точностью используются зондирующие импульсы малой скважности [1]. При этом для однозначного измерения дальности принимаются специальные меры.

Широкое распространение получили когерентно-импульсные РЛС с зондирующими импульсами высокой скважности, что обусловлено возможностью однозначного измерения дальности большого числа целей простыми средствами и с высокой разрешающей способностью. Интервал однозначного измерения доплеровской частоты $\pm 1 / 2 T$ и соответствующий ему интервал радиальной скорости оказываются недостаточными для реальных скоростей большинства радиолокационных целей. Однако в ряде РЛС, например в РЛС управления воздушным движением, метеорологических РЛС и т.п., помимо дальности требуется информация о радиальной скорости движущегося объекта.

Одним из решений данной проблемы является использование неэквидистантных когерентно-импульсных сигналов и соответствующих алгоритмов и устройств их обработки [2]. Для поступающих с чередующимися периодами повторения $T_{1}$ и $T_{2}=T_{1}-\Delta T$ отсчетов $U_{j}$, $j=\overline{1, N}$ в [3] при нечетном $N$ получены следующие алгоритмы совместного обнаружения-измерения 